\title{
XE991 and Linopirdine Are State-Dependent Inhibitors for Kv7/KCNQ Channels that Favor Activated Single Subunits ${ }^{[}$
}

\author{
Derek L Greene, Seungwoo Kang, ${ }^{1}$ and Naoto Hoshi \\ Department of Pharmacology (D.L.G., S.K., N.H.), Department of Physiology and Biophysics (N.H.), University of California Irvine, \\ Irvine, California
}

Received March 24, 2017; accepted May 5, 2017

\begin{abstract}
M-channel inhibitors, especially XE991, are being used increasingly in animal experiments; however, insufficient characterization of XE991 at times confounds the interpretation of results when using this compound. Here, we demonstrate that XE991 and linopirdine are state-dependent inhibitors that favor the activated-subunit of neuronal Kv7/KCNQ channels. We performed patch-clamp experiments on homomeric Kv7.2 or heteromeric Kv7.2/3 channels expressed in Chinese hamster ovary cells to characterize XE991 and linopirdine. Neither inhibitor was efficacious around the resting membrane potential of cells in physiologic conditions. Inhibition of Kv7.2 and Kv7.2/3 channels by XE991 was closely related with channel activation. When the voltage dependence of activation was left-shifted by retigabine or right-shifted by the mutation, Kv7.2(R214D), the
\end{abstract}

shift in half-activation voltage proportionally coincided with the shift in the half-effective potential for XE991 inhibition. Inhibition kinetics during XE991 wash-in was facilitated at depolarized potentials. Ten-minute washout of XE991 resulted in 30\% current recovery, most of which was attributed to surface transport of Kv7.2 channels. Linopirdine also exhibited similar inhibition characteristics, with the exception of near- complete current recovery after washout at depolarized potentials. Inhibition kinetics of both XE991 and linopirdine was not as sensitive to changes in voltage as would be predicted by open- channel inhibition. Instead, they were well explained by binding to a single activated subunit. The characteristics of XE991 and linopirdine should be taken into account when these M-channel inhibitors are used in experiments.

\section{Introduction}

Subclasses of Kv7/KCNQ subunits form tetrameric channels that underlie the M-current, a low-threshold noninactivating voltage-gated potassium current, which regulates neuronal excitability (Jentsch, 2000; Delmas and Brown, 2005; Greene and Hoshi, 2017). Activation of Gq-coupled receptors, such as muscarinic acetylcholine receptors $(\mathrm{m} 1$ and $\mathrm{m} 3$ ), suppresses the M-current and induces transient hyperexcitability in a wide range of neurons (Delmas and Brown, 2005). Accordingly, M-channel inhibitors were developed in an effort to ameliorate defective neuronal activity, such as in Alzheimer dementia. Linopirdine is one such prototypical compound that was found to have a cognitive enhancing effect in an animal model (Fontana et al., 1994); however, linopirdine did not pass phase 3 clinical trials (Rockwood et al., 1997). XE991 was developed as an improved compound with a similar chemical structure (Zaczek et al., 1998). Although no clinical trials have been conducted for this

This work was supported by the National Institutes of Health National Institutes of Neurologic Disorders and Stroke [Grant R01NS067288] to N.H.

${ }^{1}$ Current affiliation: Department of Anesthesiology, Pharmacology and Physiology, Rutigers, The State University of New Jersey, New Jersey Medical School, Newark, New Jersey.

https://doi.org/10.1124/jpet.117.241679.

S This article has supplemental material available at jpet.aspetjournals.org. compound, XE991 has been used increasingly in cell culture and animal experiments to investigate physiologic (Vetter et al., 2013; Young and Thomas, 2014; Martinello et al., 2015) and pathologic roles (Mani et al., 2013; Kay et al., 2015) of the M-current.

It has been shown that KCNQ2 gene knockout is lethal (Watanabe et al., 2000; Soh et al., 2014); however, administration of linopirdine is well tolerated in humans (Pieniaszek et al., 1995; Rockwood et al., 1997), as well as in animals for linopirdine and XE991 (Fontana et al., 1994; Zaczek et al., 1998). In addition, even though XE991 is a highly potent inhibitor, it sometimes requires prolonged incubation to inhibit (Yue and Yaari, 2004), or it may have no observed effect (Romero et al., 2004). These lines of evidence suggest that there are conditions in which these Kv7 channel inhibitors are not efficacious.

Since XE991 is among the predominant choices for inhibiting Kv7 channels, inconsistencies in the literature denote a need to further characterize its mode of inhibition. To this end, we performed an electrophysiological study in a heterologous expression system. We determined the conditions under which these compounds are efficacious, as well as their mode of interaction, and addressed the past inconsistencies regarding the washout of these compounds. The determined characteristics of XE991 and linopirdine should be pertinent for experimenters using these inhibitors.

ABBREVIATIONS: $\mathrm{CHO}$, Chinese hamster ovary; $K_{\mathrm{d}}$, inhibition constant; $k_{\mathrm{on}}$, association rate constant; $k_{\text {off }}$, dissociation rate constant; $\tau$, time constant; $\mathrm{mCit}$, monomeric citrine; TIRF, total internal reflection florescence. 


\section{Materials and Methods}

Reagents and Plasmids. Linopirdine (1,3-dihydro-1-phenyl-3,3bis(4-pyridinylmethyl)-2H-indol-2-one dihydrochloride), XE991 dihydrochloride (10,10-bis(4-pyridinylmethyl)-9(10H)-anthracenone dihydrochloride), and Exo1 (2-[(4-fluorobenzoyl)amino]-benzoic acid methyl ester) were purchased from Tocris (Bristol, UK). Retigabine (ethyl N-[2-amino-4-[(4-fluorophenyl)methylamino]phenyl]carbamate) was purchased from Alomone Laboratories (Jerusalem, Israel). Concanavalin A was purchased from Sigma-Aldrich (St. Louis, MO). Mammalian expression plasmids containing rat Kv7.2, Kv7.3 (Hoshi et al., 2003) and Kv7.2-monomeric citrine (mCit) (Kosenko et al., 2012) have been described. Kv7.2 mutation (R214D) was generated using QuickChange II XL site-directed mutagenesis (Agilent Technologies, San Diego, CA) and was validated by DNA sequencing.

Cell Culture. Chinese hamster ovary (CHO) hm1 cells (Jiang et al., 2015) were cultured in $\alpha$ minimum essential media containing $5 \%$ fetal calf serum and $500 \mu \mathrm{g} / \mathrm{ml} \mathrm{G418} \mathrm{sulfate.} \mathrm{Cells} \mathrm{were} \mathrm{maintained} \mathrm{in} \mathrm{a}$ humidified incubator at $37^{\circ} \mathrm{C}$ with $5 \% \mathrm{CO}_{2}$. $\mathrm{CHO}$ cells were grown to $30 \%$ confluence on 35-mm plates before being transfected with $1 \mu \mathrm{g}$ of plasmid DNA and $4 \mu \mathrm{l}$ of LT1 reagent (Mirus Bio LLC, Madison, WI).

Electrophysiology. All patch-clamp recordings were performed at room temperature on isolated $\mathrm{CHO}$ cells using an Axon Multiclamp
700B patch clamp amplifier (Molecular Devices, CA). Data were acquired using pClamp software (version 10, Molecular Devices). Signals for current traces of $\leq 1$ second duration were sampled at $2 \mathrm{kHz}$ and low-pass-filtered at $1 \mathrm{kHz}$. Current recordings between 1 and 15 seconds were sampled at $500 \mathrm{~Hz}$, and those longer than 15 seconds were sampled at $250 \mathrm{~Hz}$. Whole-cell patch-clamp recordings on $\mathrm{CHO} \mathrm{hm} 1$ cells have been described previously (Kosenko et al., 2012). Briefly, cells were constantly perfused with Q2 solution containing $144 \mathrm{mM} \mathrm{NaCl}, 5 \mathrm{mM} \mathrm{KCl}, 2 \mathrm{mM} \mathrm{CaCl}_{2}, 0.5 \mathrm{mM} \mathrm{MgCl}_{2}$, $10 \mathrm{mM}$ glucose, and $10 \mathrm{mM}$ HEPES (pH 7.4). Patch pipettes (3-4 M $\Omega$ ) were filled with intracellular solution containing $135 \mathrm{mM}$ potassium aspartate, $2 \mathrm{mM} \mathrm{MgCl}_{2}, 3 \mathrm{mM}$ EGTA, $1 \mathrm{mM} \mathrm{CaCl}_{2}, 4 \mathrm{mM}$ ATP, $0.1 \mathrm{mM}$ GTP, and $10 \mathrm{mM}$ HEPES (pH 7.2). Successful patches that maintained $\mathrm{Rs}<6 \mathrm{M} \Omega$ were selected for experiments. Liquid junction potential was not corrected in this study.

Live Cell Imaging. Protocol for total internal reflection florescence (TIRF)-based assessment of surface transport of Kv7.2 channel has been described previously (Jiang et al., 2015). Briefly, 1 day after transfection, cells were replated onto $18-\mathrm{mm}$ round cover glasses. On the 2nd day after transfection, cells were used for TIRF experiments. For recording, medium was replaced with Q2 solution. For Exo1 experiments, cells were pretreated with $100 \mu \mathrm{M}$ Exo1 in Q2 solution at
A
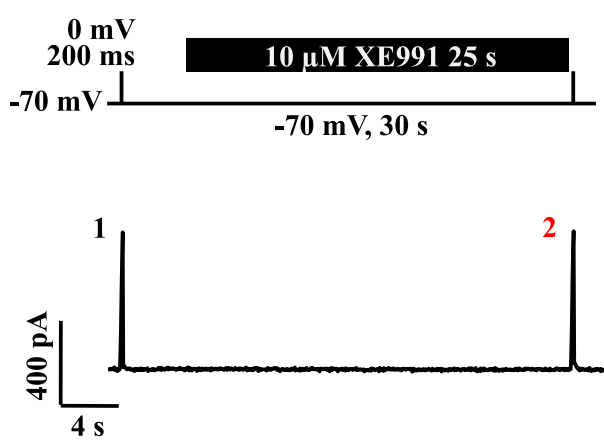

B
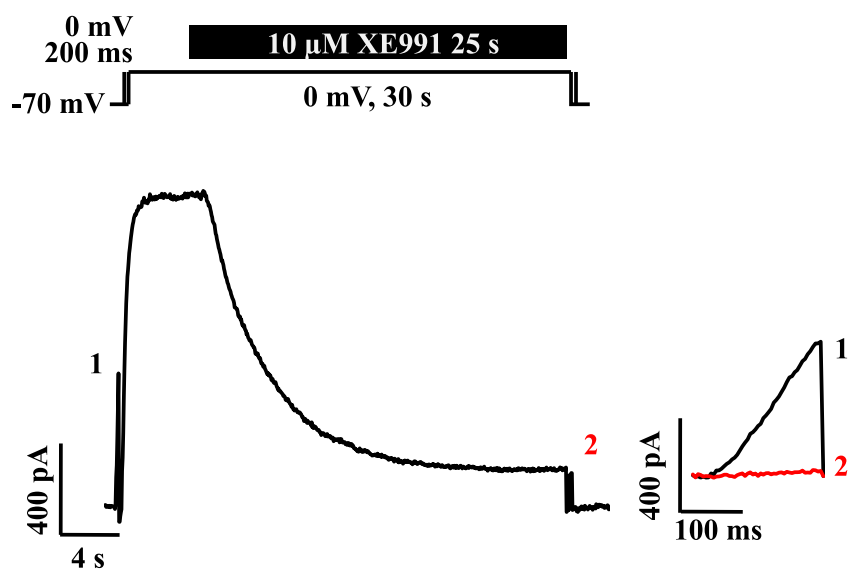

\section{Kv7.2}

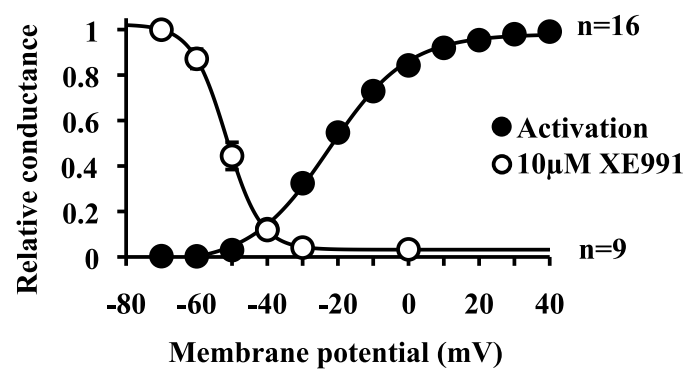

D Kv7.2/3

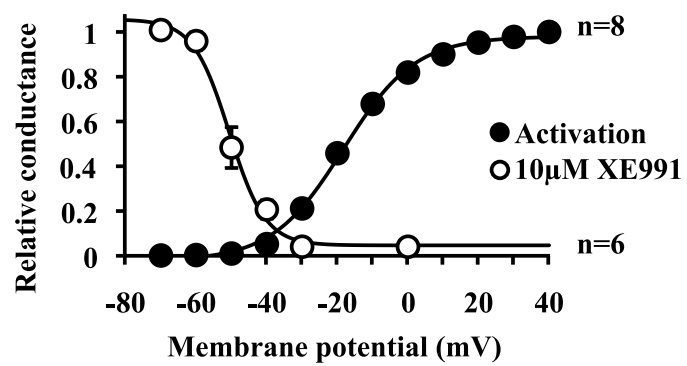

E

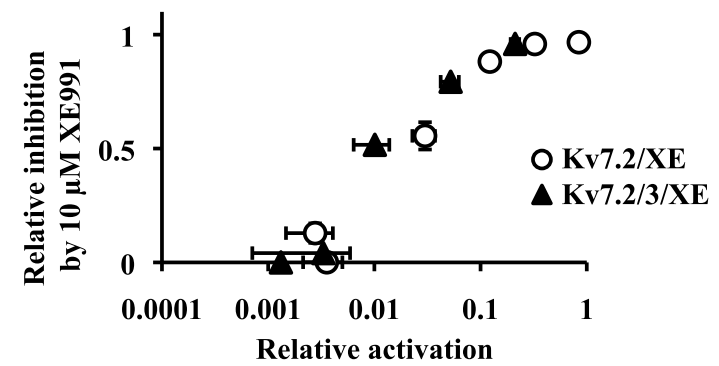

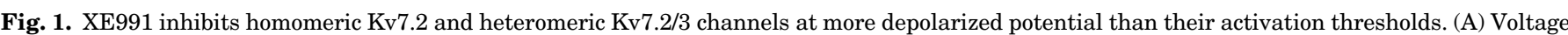

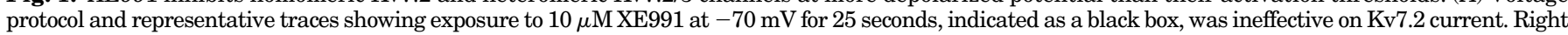

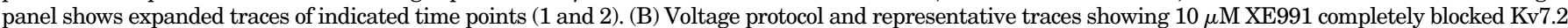

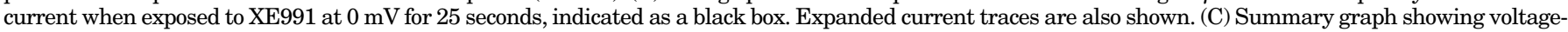

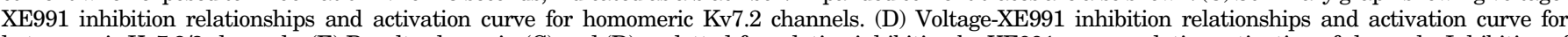

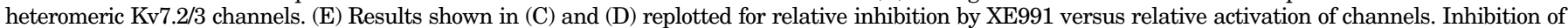

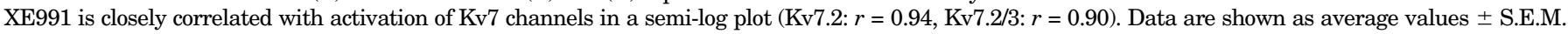


room temperature for 2 minutes before proceeding with experiments. Fluorescence emission was acquired using an inverted microscope IX-81 (Olympus Tokyo, Tokyo, Japan) with an ImageEM CCD camera (Hamamatsu Photonics, Hamamatsu, Shizuoka, Japan) controlled by MetaMorph 7.6.3 (Molecular Devices). For excitation in TIRF experiments, a 515-nm diode-pumped solid-state laser (Cobolt, Stockholm, Sweden) with an acousto-optic tunable filter was used with a TIRF module (Olympus). Emission images were obtained through a dualview module (Photometrics, Tucson, AZ) with ET535/30m, ET480/40m emission filters, and a T505lpxr dichroic mirror (Chroma Technology, Bellows Falls, VT); 100-millisecond exposure time of images was taken every 10 seconds for time-lapse imaging measurements.

Statistics. The activation curves were obtained by nonlinear regression to a Boltzmann equation, $Y=\left(1+\exp \left(\left(\mathrm{V}_{1 / 2}-\mathrm{x}\right) / \mathrm{k}\right)\right)^{-1}$, where $x$ is membrane potential, $V_{1 / 2}$ is the half activation potential, and $k$ is the slope factor unless stated otherwise in the text. All results are expressed as the mean \pm s.e.m. Statistical significance of the results was assessed by nonparametric analysis of variance (Kruskal-Wallis test) followed by Dunn's multiple comparisons test or Mann-Whitney test. All statistical tests were performed by a computer program Prism 6 (GraphPad, La Jolla, CA). $P<0.05$ is considered significant.

\section{Results}

Voltage Dependence of XE991 Inhibition is Closely Related to Activation of Kv7 Channels Rather than the Membrane Potential Per Se. During our previous study (Kay et al., 2015), we observed that XE991 is more effective in highly active neurons compared with less active neurons. It has previously been reported that M-current inhibition by XE991 is voltage-dependent; and, under some conditions, it does not inhibit the M-current (Romero et al., 2004). We alternatively reasoned that these characteristics of XE991 are derived from state-dependent inhibition rather than voltagedependent inhibition since the activation threshold of $\mathrm{Kv} 7$ channel is close to the resting membrane potential.

We characterized the voltage dependency of homomeric Kv7.2 and heteromeric Kv7.2/3 channel activation to XE991mediated inhibition. Exposure to $10 \mu \mathrm{M}$ XE991 for 25 seconds had no effects when cells were held at $-70 \mathrm{mV}$ (Fig. 1A), but it showed near-complete inhibition when XE991 was applied at potentials more positive than $-30 \mathrm{mV}$ (Fig. 1B). XE991mediated inhibition of Kv7.2 or Kv7.2/3 channels showed voltage dependence with half-inhibition potentials of $-51.6 \pm$ $0.0 \mathrm{mV}$ for Kv7.2 channels and $-50.7 \pm 0.9 \mathrm{mV}$ for $\mathrm{Kv} 7.2 / 3$ channels (Fig. 1, C and D). When XE991-mediated inhibition is plotted against relative activation of Kv7.2 and Kv7.2/3 channels (Fig. 1E), it indicates that XE991 is effective only when cells are held at potentials where Kv7 channels are activated more than $1 \%$.

To further characterize the relationships between channel activation and XE991-mediated inhibition, we shifted the activation voltage of $\mathrm{Kv} 7$ channels to more hyperpolarized potentials with retigabine and evaluated whether it changed the efficacy of XE991 (Fig. 2). In the presence of $10 \mu \mathrm{M}$ retigabine, the half-activation voltage of Kv7.2 was $-47.0 \pm$ 0.8 , a $-24-\mathrm{mV}$ shift from the control (Fig. 2B). The halfinhibition potential of XE991 also shifted $-24 \mathrm{mV}$ from the control ( $-75.2 \pm 0.6 \mathrm{mV}, n=5$ ) (Fig. 2, B and C). Notably, XE991 inhibited Kv7.2 current at a holding potential of $-70 \mathrm{mV}$ in the presence of retigabine (Fig. 2A), a potential at which XE991 did not inhibit Kv7.2 current in the control and close to the resting membrane potential in neurons (Fig. 1C).
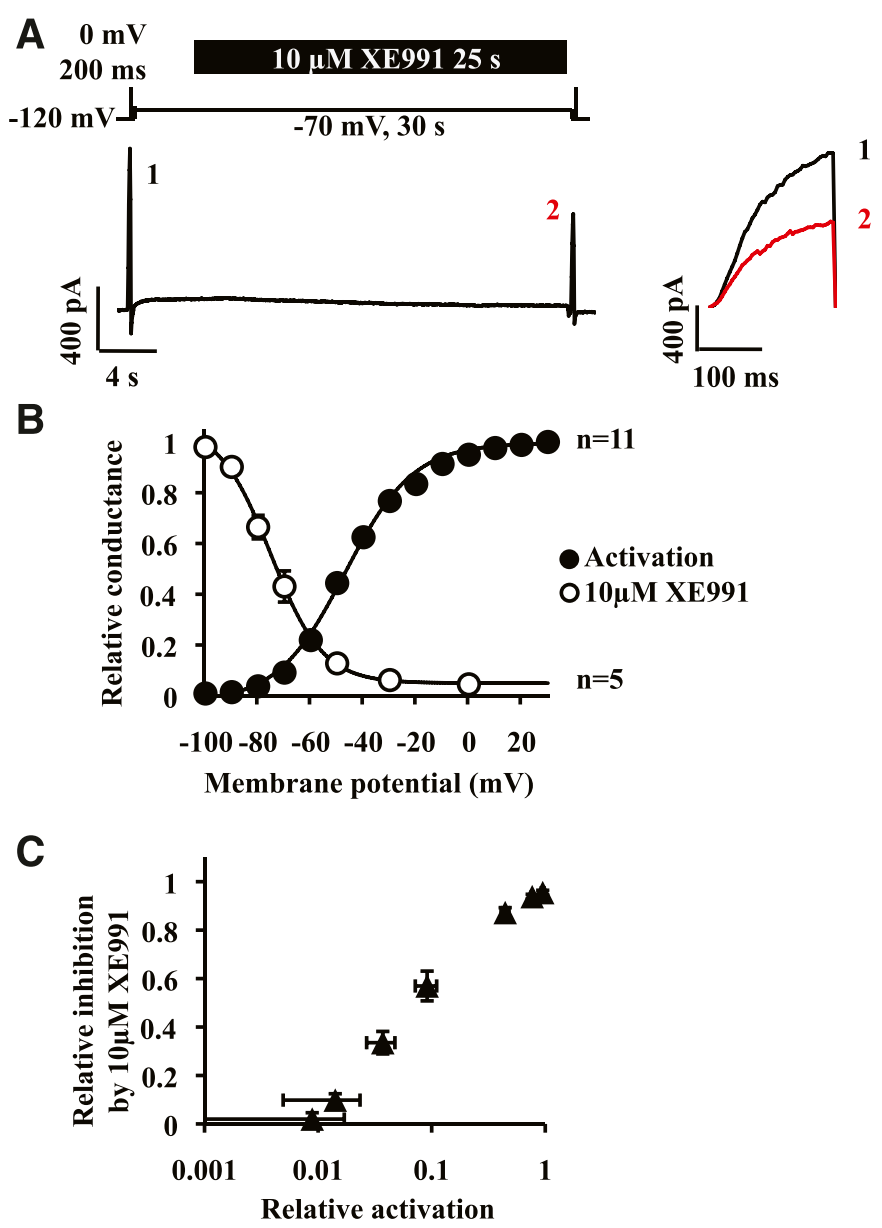

Fig. 2. Retigabine shifted effective potentials of XE991 to more negative potentials. (A) Voltage protocol and representative traces showing that when cells were pretreated with $10 \mu \mathrm{M}$ retigabine, 25-second treatment with $10 \mu \mathrm{M}$ XE991 (black box) inhibited Kv7.2 current at a holding potential of $-70 \mathrm{mV}$. (B) Voltage-XE991 inhibition relationships and activation curve for Kv7.2 channels pretreated with $10 \mu \mathrm{M}$ retigabine. (C) Activation-XE991 inhibition relationships for Kv7.2 channel from results shown in (B). Error bars show S.E.M.

An additional characterization of voltage-dependent inhibition by XE991 was assessed in a mutant Kv7.2 channel (R214D), which had a 26- $\mathrm{mV}$ right shift in the half-activation potential ( $3.4 \pm 14.0 \mathrm{mV}$ ) (Fig. 3). Accordingly, the rightward shift in the voltage dependence of activation coincided with an equivalent rightward shift $(26 \mathrm{mV})$ in the half-inhibition potential ( $-26.3 \pm 0.27 \mathrm{mV}$ ) (Fig. 3B). Relationships between inhibition by XE991 and channel activation were maintained in this condition (Fig. 3C). The voltage dependence of Kv7 channel activation versus inhibition by XE991 followed a linear function with a slope of $0.97 \pm 0.03$ compared with wildtype, retigabine-treated, and Kv7.2(R214D) channels (Fig. 3D), suggesting a close relation between the voltage dependence of Kv7.2 channel activation and the efficacy of XE991.

Inhibition Kinetics of XE991. We then tested the effect of membrane potential on inhibition kinetics (Fig. 4). First, cells were held at $-70 \mathrm{mV}$, a potential where XE991 has no effect, followed by a 20 -second depolarization to various test potentials, with or without XE991, and then analyzed the time course of inhibition. XE991 inhibited Kv7.2 currents with time constants that decreased with increasing depolarization (Fig. 4, A-C). An interesting question would be whether such 
A
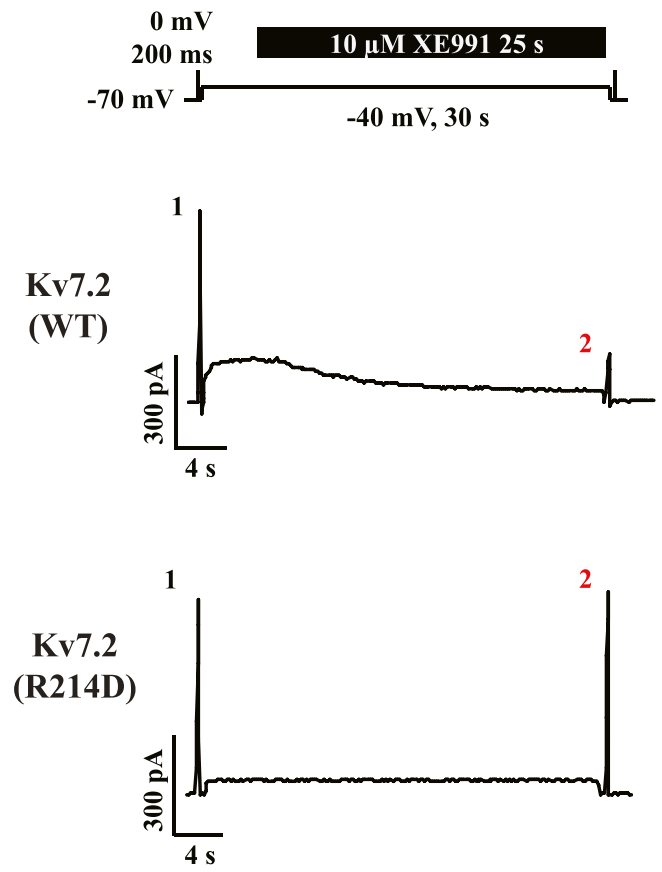

B

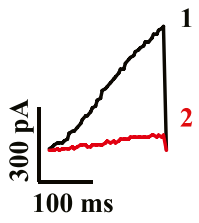

C

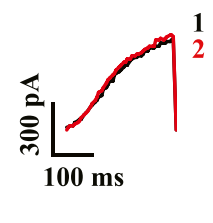

D
Kv7.2(R214D)
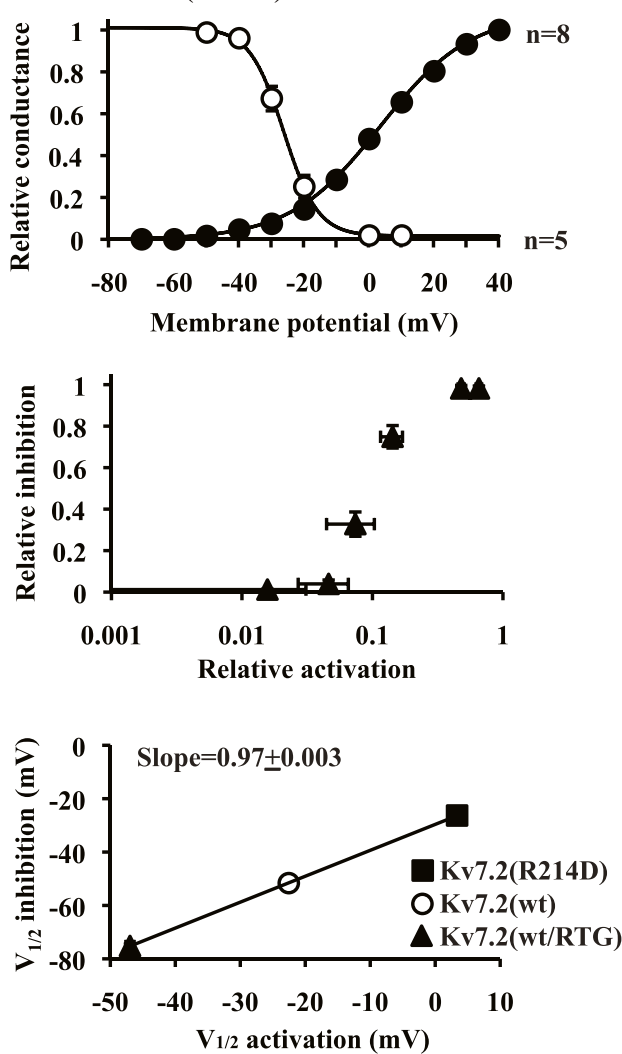

Fig. 3. Kv7.2 mutant (R214D) shifted voltage dependence of activation and XE991 inhibition to more positive potentials. (A) Voltage protocol and representative traces showing that 25-second treatment with $10 \mu \mathrm{M}$ XE991 (black box) inhibited WT Kv7.2 current at a holding potential of -40 mV, whereas Kv7.2(R214D) mutant channel had minimal inhibition at this holding potential. (B) Voltage-XE991 inhibition relationships and activation curve for Kv7.2(R214D) channels. (C) Activation-XE991 inhibition relationships for Kv7.2(R214D) channel from results shown in (B). (D) Pooled results for $\mathrm{V}_{1 / 2}$ inhibition and $\mathrm{V}_{1 / 2}$ activation potentials of $\mathrm{Kv} 7.2$, retigabine-treated Kv7.2 and Kv7.2(R214D) channel. Slope of regression line is also shown. Error bars show S.E.M.

differences in inhibition kinetics change the potency of XE991 or only the speed of inhibition. To address this question, we measured the time course of Kv7.2 current inhibition by $1 \mu \mathrm{M}$ XE991, which is close to the reported $\mathrm{IC}_{50}$ of XE991 (Wang et al., 1998), using test pulses with 20-second intervals at holding potentials of either $0 \mathrm{mV}$ or $-40 \mathrm{mV}$ (Fig. 4D). Even though there was a difference in the speed of current inhibition, depending on holding potentials, both conditions reached almost complete block after 5 minutes.

Limited Recovery of Kv7 Current after Washout of XE991 is Predominantly due to Channel Trafficking. Reports about recovery of Kv7 currents from XE991 after washout are conflicting; some groups consider inhibition by XE991 to be reversible (Rennie et al., 2001; Yue and Yaari, 2004; Zaika et al., 2006), whereas others consider it irreversible (Wladyka and Kunze, 2006; Brueggemann et al., 2012). To test this, we first treated $\mathrm{CHO}$ cells expressing Kv7.2 channels with $10 \mu \mathrm{M}$ XE991 at $0 \mathrm{mV}$ for 25 seconds, and then we held the cells at holding potentials of either -70 or $-30 \mathrm{mV}$ during the wash process to assess current recovery (Fig. 5). Washout experiments between these two potentials did not show significant difference $(20.2 \% \pm 3.9 \%$ and $18.2 \% \pm 2.9 \%$ recovery after 10 minutes of washout at $-70 \mathrm{mV}$ and $-30 \mathrm{mV}$ holding potential, respectively (Fig. 5, A and B). Control experiments without XE991 showed stable Kv7.2 current in this recording condition (Fig. 5B). We then measured rate of current recovery from distinct concentrations of XE991 (0.25,
1, $10 \mu \mathrm{M}$ ), which also showed no differences (Fig. 5C). These results suggest that current recovery after washout is quite limited for XE991. We then tested heteromeric Kv7.2/3 channels, which showed similar slow and limited recovery after washout $[33.4 \% \pm 5.9 \%(n=6)$ recovery at $-70 \mathrm{mV}, 37.8 \% \pm$ $3.8 \%(n=6)$ at $-30 \mathrm{mV}$ after 10 minutes' wash].

A $30 \%$ turnover rate of Kv7.2 channel in 10 minutes is comparable to surface transport of Kv7.2 subunit that we described previously in CHO cells (Jiang et al., 2015). To evaluate the contribution of Kv7.2 channel trafficking to the current recovery, we tested whether an exocytosis inhibitor, $100 \mu \mathrm{M}$ Exo1 (Feng et al., 2003), can suppress current recovery after washout. We first confirmed the effects of $100 \mu \mathrm{M}$ Exo1 on the surface transport of the Kv7.2 channel by total internal reflection fluorescence (TIRF) measurements using monomeric citrine-tagged Kv7.2 channel, Kv7.2-mCit. TIRF selectively illuminates $<100 \mathrm{~nm}$ from the cover glass, which can be used to monitor Kv7.2 surface transport at the bottom surface of cells (Jiang et al., 2015). To confirm Exo1 ability to suppress exocytosis, we inhibited endocytosis by $50 \mu \mathrm{g} / \mathrm{ml}$ concanavalin A, which induced a gradual increase of TIRF signal of Kv7.2-mCit owing to constitutive exocytosis (Fig. 5D) (Jiang et al., 2015). A 2-minute pretreatment with $100 \mu \mathrm{M}$ Exo1 resulted in a $75 \% \pm 5 \%$ reduction in concanavalin A-induced increase in TIRF signals from Kv7.2-mCit (Fig. 5D), confirming that most exocytosis is inhibited in this condition. Using this condition, we examined whether Exo1 
A
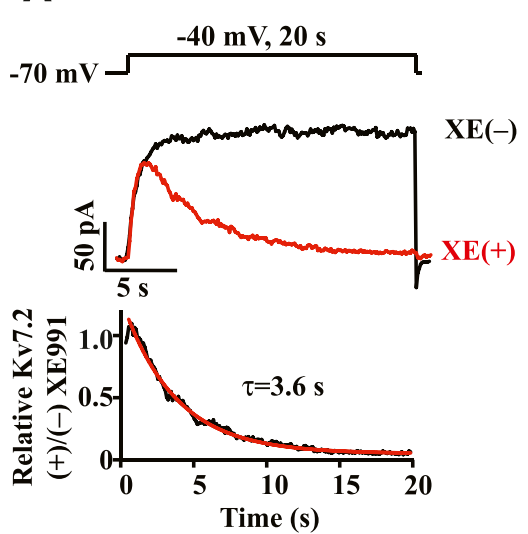

B

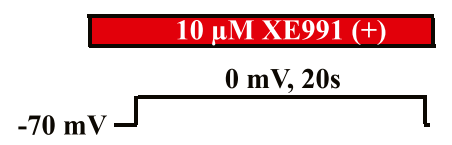

C

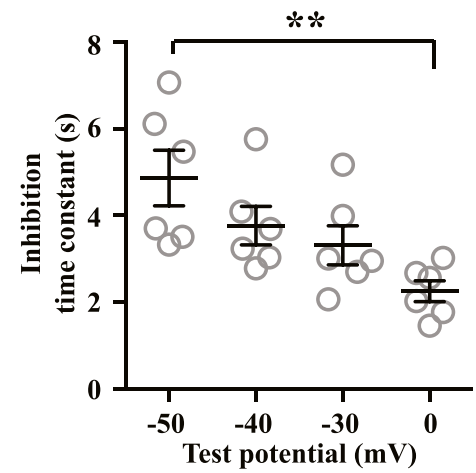

potential $(\mathrm{mV})$

D
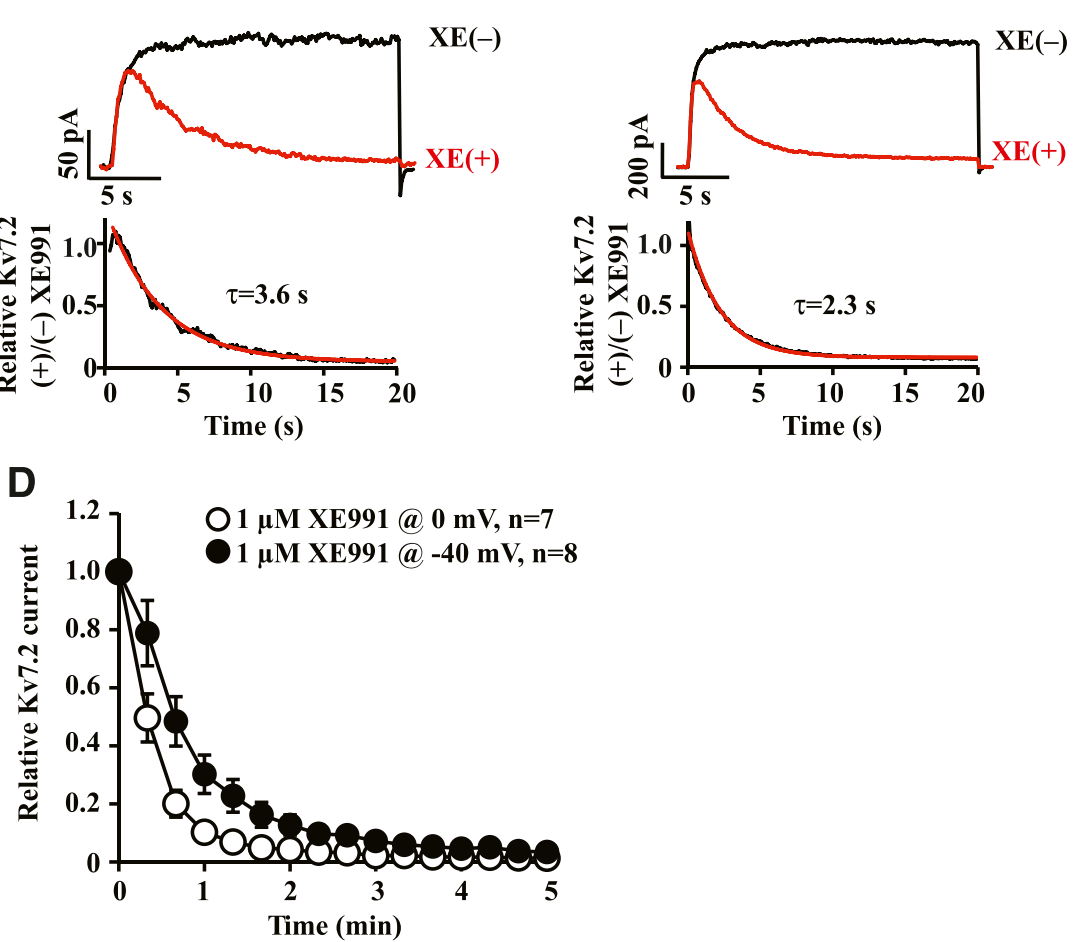


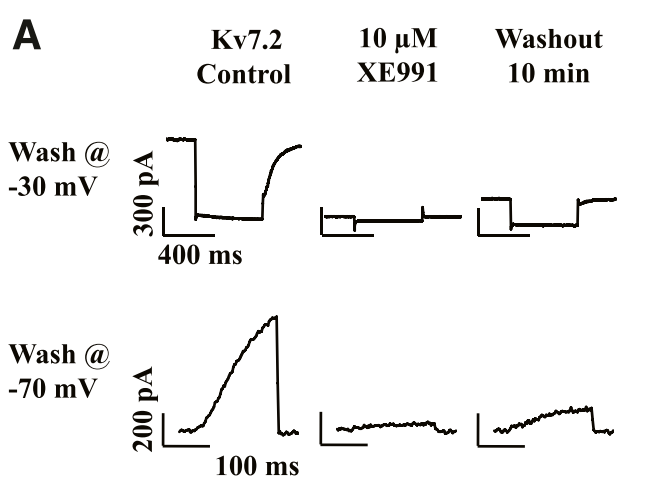

D

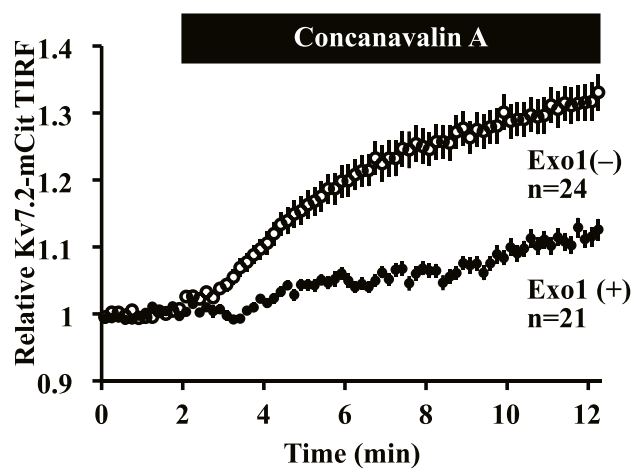

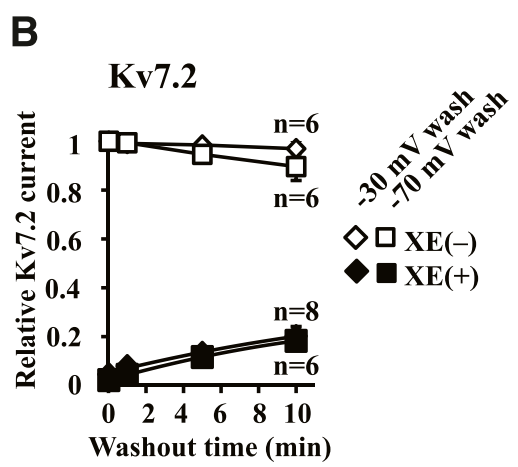

E

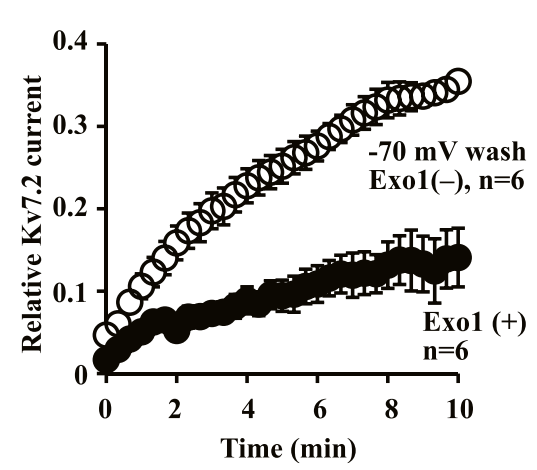

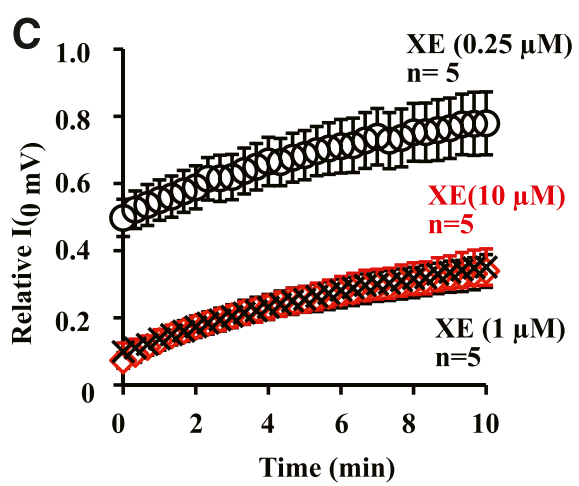

$\mathbf{F}$

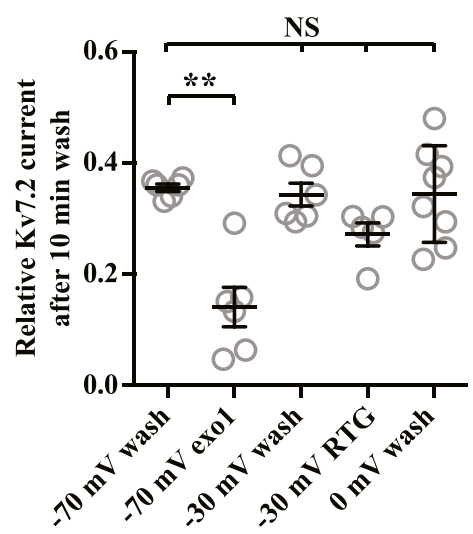

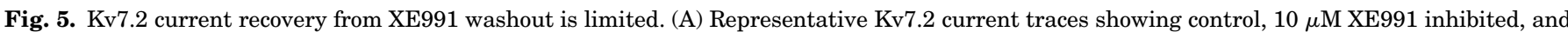

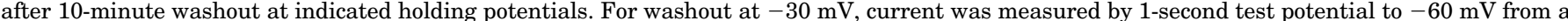

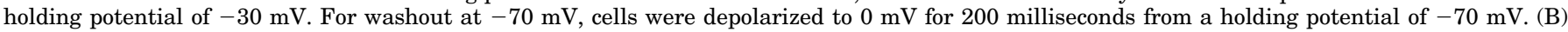

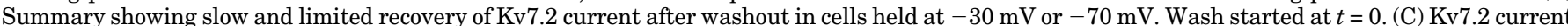

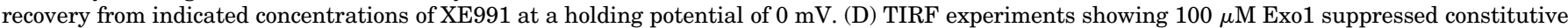

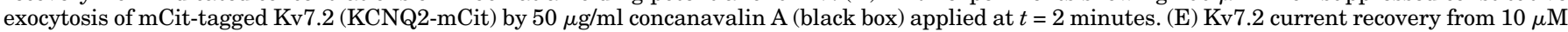

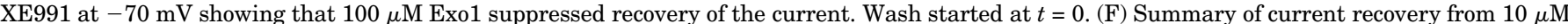

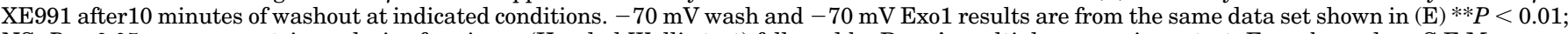
NS, $P>0.05$, nonparametric analysis of variance (Kruskal-Wallis test) followed by Dunn's multiple comparisons test. Error bars show S.E.M.

channel at the open state, then wash-in and washout time constants should show close correlation with channel activation; Namely, if inhibitors interact only with open channels, then the rate of association at the half-activation potential for Kv7.2 channel $(-21 \mathrm{mV}$, Fig. 8) should be nearly double the rate constant at maximal activation since duration of single channel opening at $V_{1 / 2}$ is half of that at maximal activation.

We assumed a single-step binding reaction for XE991 or linopirdine (inhibitors, INH), which can be described as follows in eq. 1:

$$
\begin{gathered}
\mathrm{k}_{\text {on }} \\
\mathrm{Kv7.2+ \textrm {INH }} \rightleftharpoons \mathrm{Kv} 7.2: \mathrm{INH}, \\
\mathrm{k}_{\text {off }}
\end{gathered}
$$

where $k_{\mathrm{on}}\left(\mathrm{M}^{-1} \mathrm{~s}^{-1}\right)$ is the second-order association rate constant and $k_{\text {off }}\left(\mathrm{s}^{-1}\right)$ is the first-order dissociation rate constant. Thus, this reaction can be described as follows in eq. 2 and eq. 3 :

$$
\begin{gathered}
\tau_{\text {in }}{ }^{-1}=\mathrm{k}_{\mathrm{on}}[\mathrm{INH}]+\mathrm{k}_{\mathrm{off}} \\
\tau_{\mathrm{rec}^{-1}}=\mathrm{k}_{\mathrm{off}}
\end{gathered}
$$

using the inhibition time constant $\left(\tau_{\text {in }}\right)$ and concentration of inhibitors, as described by Goldstein and Miller (1993) and
Hille (2001). In addition, $k_{\text {off }}$ can be measured directly from the rate of washout as described in eq. 3. As eq. 2 predicts, inhibition time constants are proportional to the reciprocal of the rate constant. Thus, we plotted normalized wash-in time constants to reciprocals of the activation curve $\left(\mathrm{Ch}_{\mathrm{a}}^{-1}, \mathrm{Fig} .8 \mathrm{~B}\right)$. To our surprise, neither wash-in nor washout time constants overlapped with $\mathrm{Ch}_{\mathrm{a}}^{-1}$ (Fig. 8, B and C). Instead, best-fit powers for wash-in were $0.19 \pm 0.06$ for linopirdine and $0.26 \pm 0.03$ for XE991, which were close to one-fourth power of the activation curve (Fig. $8 \mathrm{~B}, \mathrm{Ch}_{\mathrm{a}}^{1 / 4}$ ). A similar trend was observed for washout of linopirdine $(0.36 \pm 0.006$ for linopirdine washout, Fig. 8C). We did not perform this analysis for XE991 washout since it was not voltage-dependent. Considering that a single channel is composed of four subunits, a best fit to the onefourth power of channel activation suggests that binding interactions are closely related to activation of a single subunit. To evaluate this, we estimated single-subunit activation by applying the fourth-power Boltzmann equation to Kv7.2 channel activation, as shown in eq. 4 :

$$
\mathrm{Y}=\left(1+\exp \left(\left(\mathrm{V}_{1 / 2^{-}}-\mathrm{k}\right) / \mathrm{k}\right)\right)^{-4}
$$

where $\mathrm{x}$ is the membrane potential, $V_{1 / 2}$ is the half-activation potential of a single subunit, and $k$ is the slope factor. Best-fit 

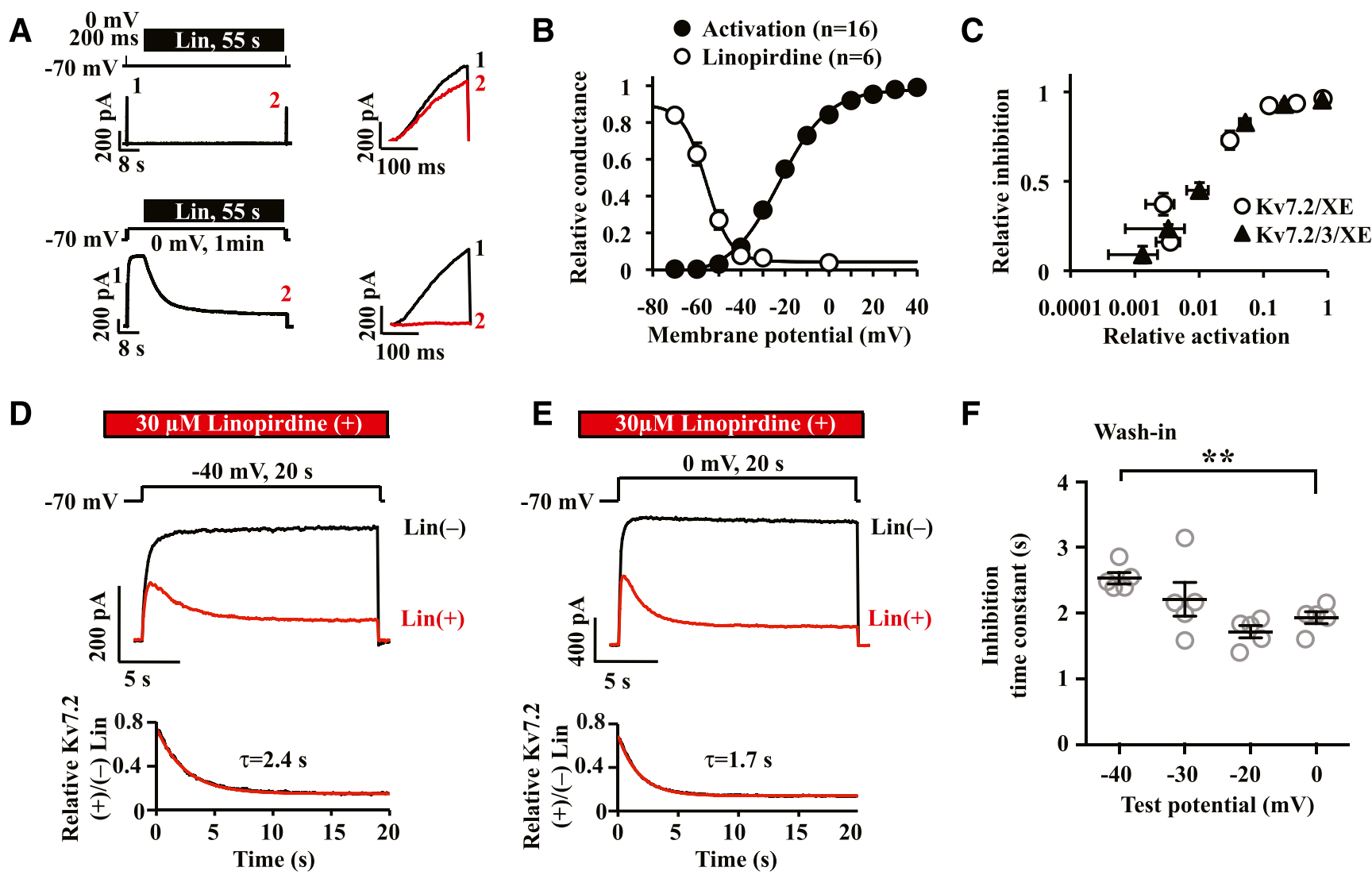

Fig. 6. Linopirdine replicated most of XE991 effects. (A) Voltage protocols and representative Kv7.2 current traces showing voltage-dependent inhibition of $30 \mu \mathrm{M}$ linopirdine (black box). Right traces show expanded currents from indicated time points 1 and 2. (B) Pooled results for voltageinhibition relationships of linopirdine and activation curve for Kv7.2 channels as measured in A. (C) Results shown in (B) replotted as relative activation against relative inhibition of Kv7.2 by linopirdine. Results of Kv7.2/3 are also included. (D) Voltage protocol and representative Kv7.2 current traces with a test potential to $-40 \mathrm{mV}$ during absence, $\operatorname{Lin}(-)$, and presence, Lin(+), of $30 \mu \mathrm{M}$ linopirdine (red box). Kv7.2 current ratio Lin(+)/Lin(-) showing that current inhibition followed a single exponential decay with the indicated time constant (bottom graph, red line). (E) Same as (D) with a test potential to $0 \mathrm{mV}$. (F) Inhibition time constants of wash-in from Kv7.2 current measured as shown in (D) and (E). As with XE991, time constants decreased with depolarizing potentials. ${ }^{* *} P<0.01$, nonparametric analysis of variance (Kruskal-Wallis test) followed by the Mann-Whitney test, $n=5$. Error bars show S.E.M.

curve for channel activation is shown as a purple curve in Fig. $8 \mathrm{~A}\left(\mathrm{Sub}_{\mathrm{a}}^{4}\right)$, and the red curve shows a derived activation curve for a single subunit ( $\left(\mathrm{Sub}_{\mathrm{a}}\right)$. Reciprocals of predicted single-subunit activation curves were also in good agreement with the inhibition kinetics of XE991 and linopirdine (Fig. 8, B and $\mathrm{C}$, red curve). Furthermore, recalculation of relationships between half-inhibition potentials of XE991 and halfactivation potentials of single-subunit of Kv7.2 channel using the results shown in Fig. 3D showed equivalent relationships (Supplemental Fig. 1).

\section{Discussion}

It has been previously reported that efficacy of linopirdine and XE991 is voltage-dependent (Romero et al., 2004). We confirmed similar voltage-dependent inhibition by these inhibitors. On the other hand, we also found that shifts in the halfinhibition potential corresponded with equivalent shifts in the half-activation voltage by retigabine or Kv7.2(R214D), which suggests that such changes in efficacy are derived from conformational changes of Kv7.2 channel subunits rather than from voltage differences across the plasma membrane. The most common class of state-dependent ion-channel inhibitor is open-channel inhibitors. Therefore, we first suspected this mechanism. In fact, transient channel activation in the presence of inhibitors, as shown in Fig. 4 and Fig. 7, is a signature profile for open-channel inhibition (Zagotta et al., 1990); however, our inhibition kinetic analysis suggests that these inhibitors bind to a single activated subunit rather than an open channel (Fig. 8, B and C).

Although the results from linopirdine mirror most of the findings from XE991, a key difference was that inhibition by linopirdine was reversible at depolarized potentials. Together with our finding from voltage-dependent wash-in kinetics, this finding suggests that linopirdine can reversibly interact with Kv7.2 channel when subunits are at the active conformation but is trapped within Kv7.2 channels when subunits are in the resting conformation after binding (Fig. 8D). Unlike linopirdine, we did not observe full recovery for XE991. Furthermore, most of the current recovery after XE991 washout was due to new channel insertion to the plasma membrane. These findings suggest that binding of XE991 to the Kv7.2 channel is apparently irreversible (Fig. 8E). On the other hand, we would like to emphasize that the Kv7.2 current can be restored from XE991-mediated inhibition as a result of channel trafficking, which explains previous observations (Rennie et al., 2001; Yue and Yaari, 2004; Zaika et al., 2006). Our results suggest $\sim 30 \%$ recovery per 10 minutes, but it may depend on cell types and experimental conditions. 
A

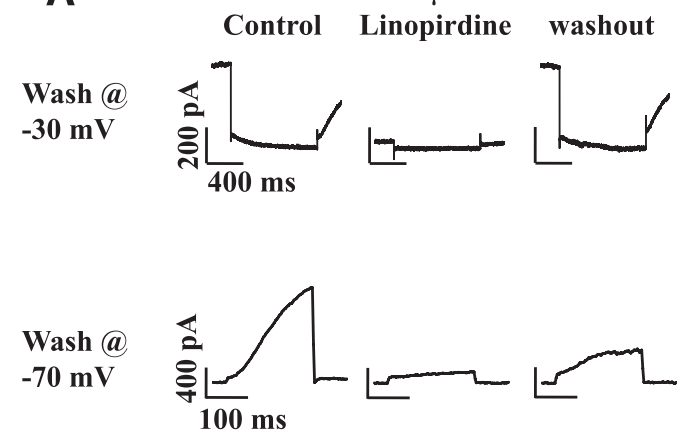

B

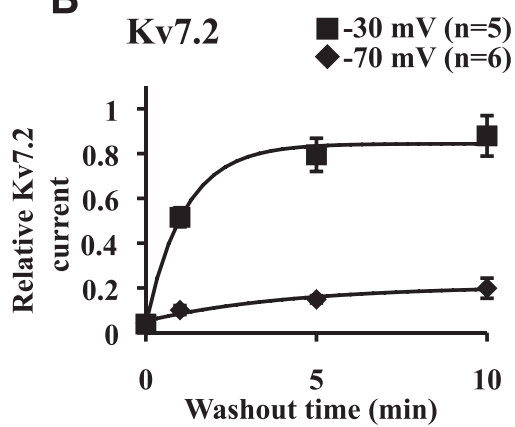

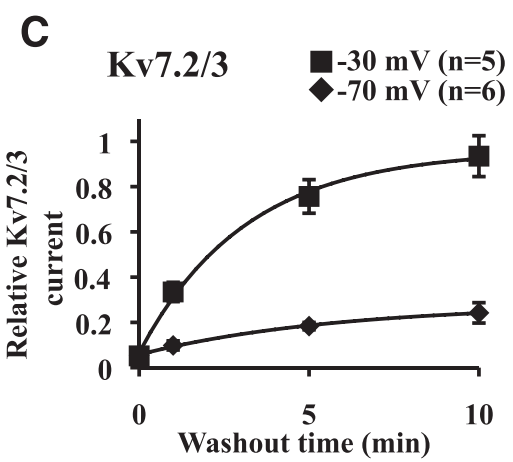

D

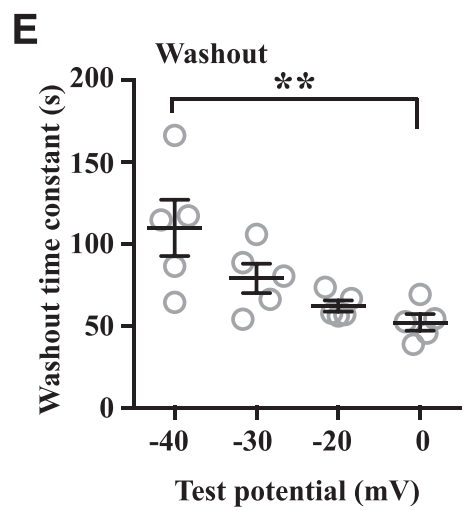

Fig. 7. Linopirdine interaction kinetics with Kv7.2 channels in relation to membrane potential. (A) Representative current traces showing Kv7.2 current recovery after washout of $30 \mu \mathrm{M}$ linopirdine from cells held at $-30 \mathrm{mV}$ or $-70 \mathrm{mV}$. For $-30 \mathrm{mV}$ holding potential during washout, Kv7.2 currents were monitored by 1-second-step hyperpolarizations to $-60 \mathrm{mV}$. For $-70 \mathrm{mV}$ holding potential, cells received a $200-\mathrm{millisecond}$ step depolarization to $0 \mathrm{mV}$. (B) Summary showing almost full recovery of Kv7.2 current when cells were washed at $-30 \mathrm{mV}$ while showing slow and limited recovery at $-70 \mathrm{mV}$. (C) Similar full recovery was observed with heteromeric Kv7.2/3 channels at $-30 \mathrm{mV}$. (D) Current recovery during washout from $30 \mu \mathrm{M}$ linopirdine at indicated holding potentials. $T=0$ indicates beginning of washout. Current was measured by a 500-millisecond-step hyperpolarization to $-60 \mathrm{mV}$ with 20 -second intervals from indicated holding potentials. (E) Summary of current recovery-time constants at indicated holding potentials during washout. ${ }^{* *} P<0.01$, nonparametric analysis of variance (Kruskal-Wallis test), followed by the Mann-Whitney test, $n=5$. Error bars show S.E.M.

Our findings in this report suggest that XE991 might not be efficacious for cells staying at resting membrane potentials, such as in silent or scarcely firing neurons. Coadministration of XE991 and retigabine would be helpful to remove such biased efficacy in animal experiments. For cultured neurons, holding at depolarized potentials during voltage-clamp experiments or coadministration of XE991 and retigabine or high potassium would be effective.

Activated subunit inhibition and slow binding kinetics of these inhibitors may explain why these compounds are well tolerated in animals (Mani et al., 2013; Vetter et al., 2013; Young and Thomas, 2014; Kay et al., 2015) without causing lethal seizures, as seen in KCNQ2 gene knockout mice (Watanabe et al., 2000; Soh et al., 2014). In addition, preferential M-channel inhibition in highly active neurons by these inhibitors would exaggerate neurotransmitter-mediated M-current suppression, which may underlie the cognitive enhancing action of these compounds.

\section{Authorship Contributions}

Participated in research design: Greene, Hoshi.

Conducted experiments: Greene, Hoshi.

Contributed new reagents: Kang.

Performed data analysis: Greene, Hoshi.

Wrote or contributed to the writing of the manuscript: Greene, Kang, Hoshi.

\section{References}

Brueggemann LI, Kakad PP, Love RB, Solway J, Dowell ML, Cribbs LL, and Byron KL (2012) Kv7 potassium channels in airway smooth muscle cells: signal transduction intermediates and pharmacological targets for bronchodilator therapy. Am J Physiol Lung Cell Mol Physiol 302:L120-L132.

Delmas P and Brown DA (2005) Pathways modulating neural KCNQ/M (Kv7) potassium channels. Nat Rev Neurosci 6:850-862.

Feng Y, Yu S, Lasell TK, Jadhav AP, Macia E, Chardin P, Melancon P, Roth M, Mitchison T, and Kirchhausen T (2003) Exo1: a new chemical inhibitor of the exocytic pathway. Proc Natl Acad Sci USA 100:6469-6474.

Fontana DJ, Inouye GT, and Johnson RM (1994) Linopirdine (DuP 996) improves performance in several tests of learning and memory by modulation of cholinergic neurotransmission. Pharmacol Biochem Behav 49:1075-1082.

Goldstein SA and Miller C (1993) Mechanism of charybdotoxin block of a voltagegated K+ channel. Biophys J 65:1613-1619.

Greene DL and Hoshi N (2017) Modulation of Kv7 channels and excitability in the brain. Cell Mol Life Sci 74:495-508.

Hille B (2001) Ion Channels of Excitable Membranes, Sinauer Associates, Inc., Sunderland, MA.

Hoshi N, Zhang JS, Omaki M, Takeuchi T, Yokoyama S, Wanaverbecq N, Langeberg LK, Yoneda Y, Scott JD, Brown DA, et al. (2003) AKAP150 signaling complex promotes suppression of the M-current by muscarinic agonists. Nat Neurosci 6 : $564-571$.

Jentsch TJ (2000) Neuronal KCNQ potassium channels: physiology and role in disease. Nat Rev Neurosci 1:21-30.

Jiang L, Kosenko A, Yu C, Huang L, Li X, and Hoshi N (2015) Activation of m1 muscarinic acetylcholine receptor induces surface transport of KCNQ channels through a CRMP-2-mediated pathway. J Cell Sci 128:4235-4245.

Kay HY, Greene DL, Kang S, Kosenko A, and Hoshi N (2015) M-current preservation contributes to anticonvulsant effects of valproic acid. $J$ Clin Invest 125: 3904-3914.

Kosenko A, Kang S, Smith IM, Greene DL, Langeberg LK, Scott JD, and Hoshi N (2012) Coordinated signal integration at the M-type potassium channel upon muscarinic stimulation. EMBO $J$ 31:3147-3156.

Mani BK, O'Dowd J, Kumar L, Brueggemann LI, Ross M, and Byron KL (2013) Vascular KCNQ (Kv7) potassium channels as common signaling intermediates and therapeutic targets in cerebral vasospasm. $J$ Cardiovasc Pharmacol 61:51-62. 


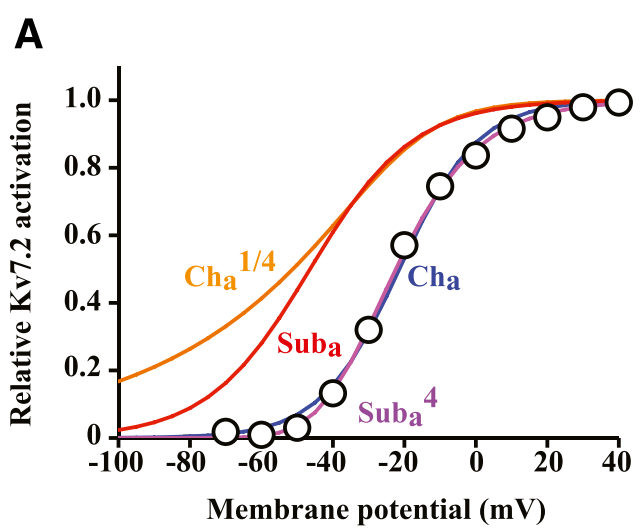

D

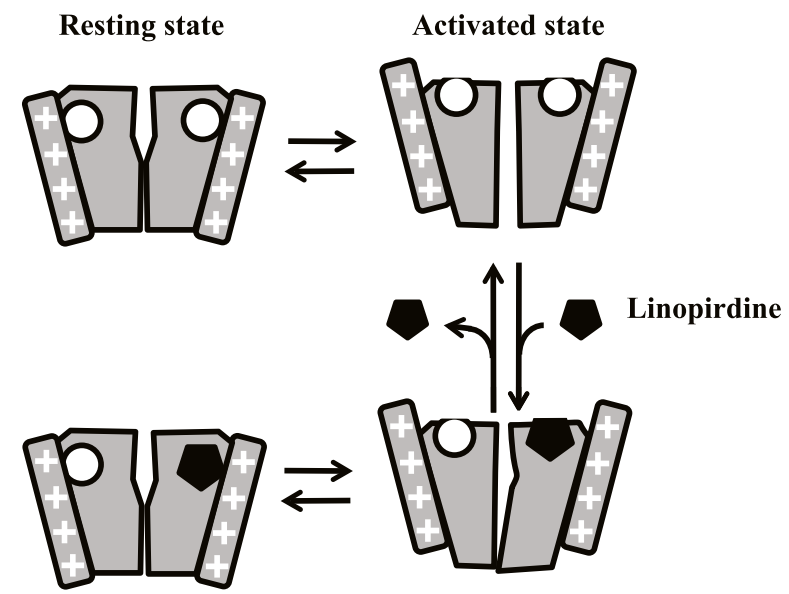

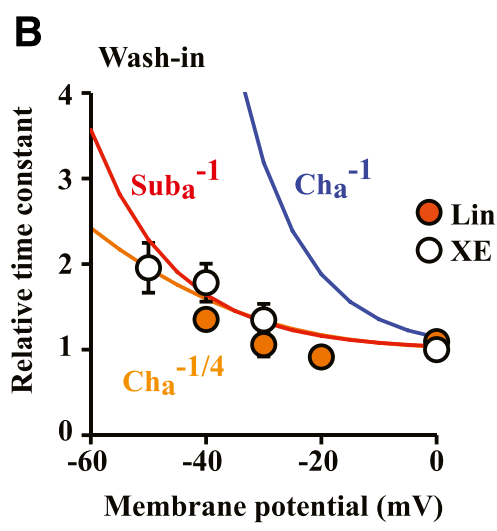

$\mathbf{E}$

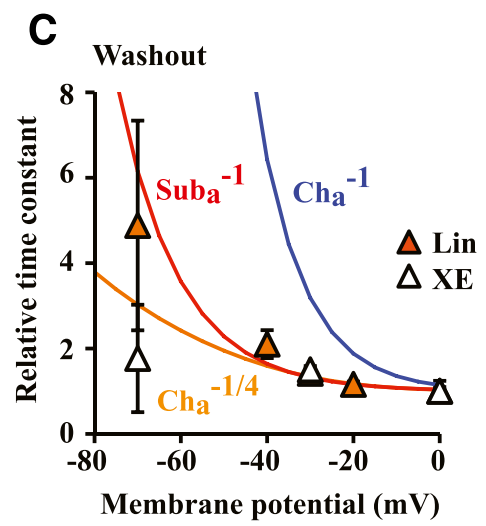

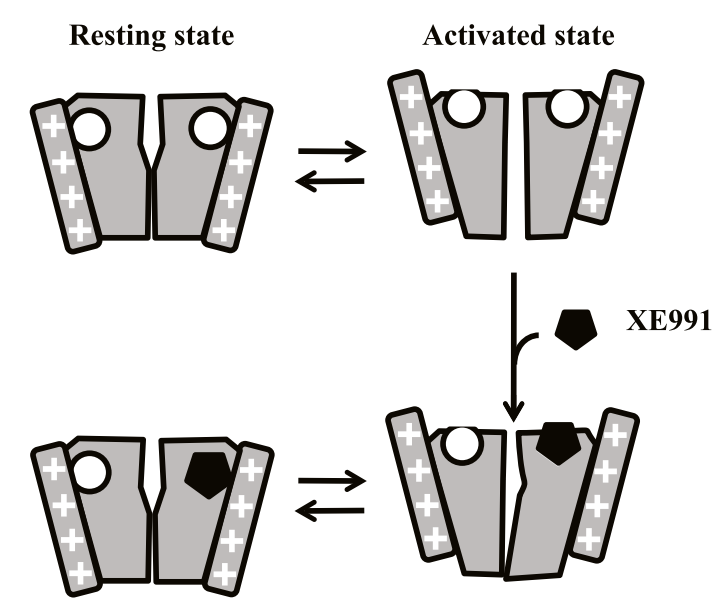

Fig. 8. XE991 and linopirdine interaction with Kv7.2 relates with activation of single subunit. (A) Activation curve for Kv7.2 channel (blue curve, Cha), and one-quarter power of its Boltzmann function (orange curve, $\mathrm{Ch}_{\mathrm{a}}^{1 / 4}$ ). Activation curve for a single subunit (red curve, Sub ${ }_{\mathrm{a}}$ ) and its fourth power function (purple curve, $\mathrm{Sub}_{\mathrm{a}}^{4}$ ) are also shown (see text for details). (B) Relative inhibition time constants calculated from Fig. 4C (XE991, open circle) and Fig. 7C (linopirdine, orange circle) normalized to those from $0 \mathrm{mV}$ plotted as a function of voltage. The reciprocal of the activation curves of $\mathrm{Ch}_{\mathrm{a}}$, $\mathrm{Ch}_{\mathrm{a}}^{1 / 4}$ and $\mathrm{Sub}_{\mathrm{a}}$ shown in (A) are also shown. (C) Relative recovery time constants from experiments shown in Fig. 4 and Fig. 5B (XE991, open triangle) and Fig. 6E and $7 \mathrm{E}$ (linopirdine, orange triangle), overlaid with the reciprocal of the activation curves of $\mathrm{Ch}_{\mathrm{a}}, \mathrm{Ch}_{\mathrm{a}} \mathrm{i}_{4}$, and Sub $\mathrm{a}$. (D) Schematic model for interaction between linopirdine and Kv7.2 channel. (E) Schematic model for interaction between XE991 and Kv7.2 channel. Error bars show S.E.M.

Martinello K, Huang Z, Lujan R, Tran B, Watanabe M, Cooper EC, Brown DA, and Shah MM (2015) Cholinergic afferent stimulation induces axonal function plasticity in adult hippocampal granule cells. Neuron 85:346-363.

Pieniaszek, Jr HJ, Fiske WD, Saxton TD, Kim YS, Garner DM, Xilinas M, and Martz R (1995) Single-dose pharmacokinetics, safety, and tolerance of linopirdine (DuP 996) in healthy young adults and elderly volunteers. J Clin Pharmacol 35:22-30.

Rennie KJ, Weng T, and Correia MJ (2001) Effects of KCNQ channel blockers on $\mathrm{K}(+)$ currents in vestibular hair cells. Am J Physiol Cell Physiol 280: C473-C480.

Rockwood K, Beattie BL, Eastwood MR, Feldman H, Mohr E, Pryse-Phillips W, and Gauthier S (1997) A randomized, controlled trial of linopirdine in the treatment of Alzheimer's disease. Can J Neurol Sci 4(2):140-145.

Romero M, Reboreda A, Sánchez E, and Lamas JA (2004) Newly developed blockers of the M-current do not reduce spike frequency adaptation in cultured mouse sympathetic neurons. Eur J Neurosci 19:2693-2702.

Soh H, Pant R, LoTurco JJ, and Tzingounis AV (2014) Conditional deletions of epilepsy-associated KCNQ2 and KCNQ3 channels from cerebral cortex cause differential effects on neuronal excitability. J Neurosci 34:5311-5321.

Vetter I, Hein A, Sattler S, Hessler S, Touska F, Bressan E, Parra A, Hager U, Leffler A, Boukalova S, et al. (2013) Amplified cold transduction in native nociceptors by M-channel inhibition. J Neurosci 33:16627-16641.

Wang HS, Pan Z, Shi W, Brown BS, Wymore RS, Cohen IS, Dixon JE, and McKinnon D (1998) KCNQ2 and KCNQ3 potassium channel subunits: molecular correlates of the M-channel. Science 282:1890-1893.
Watanabe H, Nagata E, Kosakai A, Nakamura M, Yokoyama M, Tanaka K, and Sasai H (2000) Disruption of the epilepsy KCNQ2 gene results in neural hyperexcitability. $J$ Neurochem 75:28-33.

Wladyka CL and Kunze DL (2006) KCNQ/M-currents contribute to the resting membrane potential in rat visceral sensory neurons. $J$ Physiol 575:175-189.

Young MB and Thomas SA (2014) M1-muscarinic receptors promote fear memory consolidation via phospholipase C and the M-current. J Neurosci 34:1570-1578.

Yue C and Yaari Y (2004) KCNQ/M channels control spike afterdepolarization and burst generation in hippocampal neurons. J Neurosci 24:4614-4624.

Zaczek R, Chorvat RJ, Saye JA, Pierdomenico ME, Maciag CM, Logue AR, Fisher BN, Rominger DH, and Earl RA (1998) Two new potent neurotransmitter release enhancers, 10,10-bis(4-pyridinylmethyl)-9(10H)-anthracenone and 10,10-bis(2-fluoro-4 pyridinylmethyl)-9(10H)-anthracenone: comparison to linopirdine. J Pharmacol Exp Ther 285:724-730.

Zagotta WN, Hoshi T, and Aldrich RW (1990) Restoration of inactivation in mutants of Shaker potassium channels by a peptide derived from ShB. Science 250:568-571.

Zaika O, Lara LS, Gamper N, Hilgemann DW, Jaffe DB, and Shapiro MS (2006) Angiotensin II regulates neuronal excitability via phosphatidylinositol 4,5-bisphosphatedependent modulation of Kv7 (M-type) K+ channels. J Physiol 575:49-67.

Address correspondence to: Naoto Hoshi, Department of Pharmacology, University of California, Irvine, 360 Med Surge II, Irvine, CA 92697. E-mail: nhoshi@uci.edu 